\title{
EFFECT OF FATTY ACID-BASED FUNCTIONAL LIPIDOMICS IN WOMEN WITH BREAST CANCER
}

\author{
PREETHIKA A ${ }^{1}$, SUCHETHA KUMARI N²*
}

${ }^{1}$ Research Scholar, K S Hegde Medical Academy, Mangaluru, Karnataka. ${ }^{2}$ Department of Biochemistry, K S Hegde Medical Academy, Mangaluru, Karnataka. Email: suchethakumari2018@gmail.com

Received: 12 July 2019, Revised and Accepted, 10 October 2019

ABSTRACT

Objective: Lipid metabolism alteration is a prominent feature during malignant transformation. We investigated the difference in plasma fatty acids (FAs) and molecular biomarkers in breast cancer women and controls.

Methods: This comparative study was carried out on eighty breast cancer women and forty control women. FAs were analyzed in gas chromatography and calculated as a percentage of total FAs. FA molecular markers were estimated by the FA data. Non-parametric statistical tests were used.

Results: Saturated FAs were higher in the case group. Control group had higher linoleic acid ( $\mathrm{p}=0.04)$, suggesting decreased desaturase activity. N-6 FAs were higher and n-3 FAs were lower in breast cancer cases. The n6/n3 ratio, cardiovascular risk ratio (p<0.001), and inflammatory risk ratio were high, whereas saturation index and unsaturation index $(\mathrm{p}=0.05)$ were lower in breast cancer cases.

Conclusion: Lipidomics of the cell membrane is significantly influenced by FAs, and the dietary FAs regulate the enzymatic activities in the FA metabolism pathway.

Keywords: Functional lipidomics, Fatty acids, Breast cancer, Cell membrane fatty acid profile, Fatty acid profile.

(C) 2019 The Authors. Published by Innovare Academic Sciences Pvt Ltd. This is an open access article under the CC BY license (http://creativecommons. org/licenses/by/4. 0/) DOI: http://dx.doi.org/10.22159/ajpcr.2019.v12i10.34888

\section{INTRODUCTION}

Breast cancer is a common form of cancer leading to death globally. Multiple factors such as lifestyle factors, genetic variation, dietary factors, and toxic environmental factors affect breast cancer development and proliferation in different population [1,2]. Regarding diet, fatty acids (FAs) have drawn attention in recent years, due to their role in cellular membrane integrity.

The difference in the lipid pathway in normal and cancer cells makes a target for cancer therapeutics. Lipidomics study is attracting a growing interest in cancer, mainly due to its effect on polyunsaturated fatty acids(PUFAs) metabolism in breast cancer cells. Enzymes such as delta 5 Desaturase (D5D), delta 6 desaturase (D6D), delta 4 desaturase, and elongases are required for the synthesis of PUFAs, encoded in the FA desaturase genes. Studies have shown that n- 6 FAs are proinflammatory, whereas n-3 FAs exert anti-inflammatory property [3-5].

A recent advance on functional lipidomics has shown how the lipids alter the signaling pathway at the molecular level [6]. The FA profile and its interaction depend on different factors such as gene, metabolism, and diet, to maintain homeostasis in an individual. Functional lipidomics represents the investigation of FA-based functional and structural role on the cell membrane and changes in vivo. This helps to connect changes with biological significance.

The objective of this study was to examine the effects of plasma FAs on the cell membrane of breast cancer patients and to evaluate in terms of functional lipidomics.

\section{METHODS}

\section{Study population}

This comparative study involved eighty breast cancer women within the age group of 25-60 years and forty age-matched control women. Women who underwent a clinical examination and histopathologically proven women with breast tumor were included in the study. Women who are pregnant, who had undergone breast implants, and those with a previous history of other tumors were excluded from the study. Controls were recruited based on the following criteria: those who attend the oncology outpatient department and had not undergone breast-conserving surgery/mastectomy/not reported any history of cancer at the time of case diagnosis. The study was conducted after ethical approval and obtaining written informed consent from the recruited women. Blood samples collected from the women were centrifuged to collect plasma and stored as aliquots at $-20^{\circ} \mathrm{C}$.

\section{Analysis of plasma FAs}

Lipids were extracted using $100 \mu \mathrm{l}$ plasma and converted into FA methyl esters (FAMEs) using Metcalfe et al.'s method [7]. The FAMEs were analyzed using gas chromatography (Agilent 7820A) equipped with J\&W DB-23 column. Triheptadecanoin (NuChek Prep, Elysian, MN, USA) was used as an internal standard. Elution times of the commercially available standard FAs (Supelco 37 component FAME mix, Sigma Aldrich) were used as a reference and compared to identify the peaks. Individual plasma FAs were expressed as a percentage of total FAs present.

\section{Plasma FA profile}

The FA panel included saturated FA (SFA-palmitic acid: C16:0; stearic acid: C18:0); monounsaturated FAs (MUFAs) (palmitoleic acid: C16:1 and oleic acid (OA): C18:1 n-6 PUFAs (linoleic acid (LA): Gamma-linolenic acid (GLA): C18:2; dihomo- $\gamma$-LA (DGLA): 20:3; arachidonic acid (ARA): C20:4) and n-3 PUFAs (alpha-linolenic acid: C18:3; eicosapentaenoic acid (EPA):C20:5; Docosahexaenoic acid (DHA): C22:6).

Enzymatic indexes of desaturase enzymes involved in the PUFA pathway were calculated by the product/precursor ratio of the individual FAs.

D5D activity= ARA/DGLA; D5D enzymes catalyze the conversion of DGLA to AA.

D6D activity= GLA/LA; D6D converts LA to GLA 
Molecular biomarkers (FA indexes) $[8,9]$

Saturation Index $(\mathrm{SI})=\% \mathrm{SFA} / \% \mathrm{MUFA}$ : It regulates the cell membrane fluidity. The higher the SI, the lower the membrane fluidity. The reference range is $1.7-2.0$.

Inflammatory Risk Index $=\%$ n-6 PUFA) $/ \%$ n-3 PUFA. The ratio shows the lipid pathway to form pro-inflammatory or anti-inflammatory mediators. Reference range $=3.5-5.5$

Cardiovascular disease (CVD) Risk Index $=\% \mathrm{EPA}+\% \mathrm{DHA}$. The risk can be categorized into high risk $<4 \%$, intermediate $4-8 \%$, and low risk $>8 \%$.

PUFA balance ((\%EPA + \%DHA)/total PUFA*100), UnsaturationIndex $(U I)=((\%$ MUFA $* 1)+(\%$ LA $* 2)+(\%$ DGLA $* 3)+(\% A A * 4)+(\%$ EPA $* 5)+(\%$ DHA *6), and Peroxidation Index (PI) $=((\%$ MUFA *0.025) + $\left.\left(\% \mathrm{LA}^{*} 1\right)+(\% \mathrm{DGLA} * 2)+(\% \mathrm{AA} * 4)+(\% \mathrm{EPA} * 6)+(\% \mathrm{DHA} * 8)\right)$.

\section{Statistical analysis}

Variables were represented as median (interquartile range) and percentage. Mann-Whitney U-test was used to compare between the groups. All $\mathrm{P}$ values were two sided with a significance of $\mathrm{p} \leq 0.05$. Statistical Package for the Social Sciences (SPSS V.16.0; IBM Corp) was used for the analysis.

\section{RESULTS}

A total of 120 women were recruited for the study. The groups were similar in terms of age and body mass index (BMI). Characteristics of the study groups are summarized in Table 1.

\section{FA profile}

Table 2 summarizes the FA profile among the groups. Total SFAs did not vary among the case and control groups. MUFAs, palmitoleic acid, and $\mathrm{OA}$ were higher in the case group. The palmitoleic percentage was statistically significant $(\mathrm{p}=0.005)$. The percentage of $n-6$ FAs was higher in the case group except for DGLA. The percentage of LA among the group differed statistically significantly $(p=0.04)$. Among the $n-3$ FAs, DPA was higher in the case group, and the statistical difference was observed in the EPA percentage $(\mathrm{p}=0.02)$. No much difference was observed in desaturase activity among the groups, suggesting similar activity among the groups.

\section{Molecular biomarkers}

Membrane Fluidity Index: SFA/MUFA ratio was lower in the case group and differed statistically significantly $(0.51$; range: $0.41-2.95, \mathrm{p}=0.024)$ (Fig. 1).

Inflammatory Risk Index: The $\mathrm{n} 6 / \mathrm{n} 3$ ratio was 5.5 in the control and 6.9 in the case groups. Case group showed a higher value than the reference range, but no statistical difference was observed among the groups.

CVD Risk Index: The n-3 CVD risk ranged between 4\% and 8\% (intermediate risk) in control group [1.6 (0.98-2.280], whereas in breast cancer cases, it was at risk group with $2.7 \%(1.18$ [0.65-1.64]; $\mathrm{p}<0.001)$.

PUFA balance was higher in the control group (0.074). UI was higher in the cancer group $(\mathrm{p}=0.05)$, no much differences were observed in PI among the groups.

Saturation Index = SFAs $/$ MFAs; Inflammatory Risk Index = $\%$ n-6PUFA $/ \%$ n-3 PUFA: Cardiovascular disease (CVD) Risk Index = (\%EPA + \%DHA); PUFA balance ((\%EPA + \%DHA)/total PUFA*100), UnsaturationIndex $(\mathrm{UI})=\left(\left(\% \mathrm{MUFA}^{*} 1\right)+(\% \mathrm{LA} * 2)+(\% \mathrm{DGLA} * 3)+(\% \mathrm{AA} * 4)+(\% \mathrm{EPA} * 5)\right.$ $+(\% \mathrm{DHA} * 6)$, and Peroxidation Index $(\mathrm{PI})=((\%$ MUFA $* 0.025)+(\% \mathrm{LA}$ $* 1)+(\% \mathrm{DGLA} * 2)+(\% \mathrm{AA} * 4)+(\% \mathrm{EPA} * 6)+(\% \mathrm{DHA} * 8))$.

\section{DISCUSSION}

The functional lipidomics was used to interpret the effects of dietary FAs in patients with breast cancer. The metabolic changes in tumor involve carbohydrates, protein, and lipid metabolism for cancer cell proliferation. A high carbohydrate level with increased insulin levels promotes de novo synthesis of triglycerides and lipids for lipid storage in the liver and adipose tissue. The de novo synthesis of lipids is emphasized in cancer cells, as they present high levels of lipogenesis and increased requirement of the unsaturated FAs for survival under adverse conditions $[10,11]$.

We found increased MUFAs in cancer women compared to controls, indicating increased activity of elongases and desaturases thereby lowering the saturation index and increasing the membrane fluidity. This indicates the significance of desaturation in cell membrane fluidity and permeability. Poor membrane fluidity compromises with the stability of the cell membrane, leading to peroxidation and cell damage $[12,13]$. Few studies propose increased MUFA production by the cancer cells guarantees cell survival by altering the fluid state $[14,15]$.

Palmitoleic acid level was significant in cancer cases, which is a biomarker for obesity. In cancer, palmitoleic acid level helps to provide FAs in cell membrane phospholipids [8]. LA was higher in the control group. It is the second-highest FA available in the body next to OA. High LA levels were associated with low AA levels, suggesting low activity of the D6D enzymes when compared to cancer cells. Increased AA levels and low LA in breast cancer cells can be due to the insulin stimulation of the desaturase enzymes in the n-6 pathway [9].

Table 1: Characteristics of the control and breast cancer cases

\begin{tabular}{|c|c|c|}
\hline Characteristics & Controls $(n=40)$ & Cases $(n=80)$ \\
\hline $\begin{array}{l}\text { Mean age (years) at blood } \\
\text { collection }\end{array}$ & $43( \pm 7)$ & $45( \pm 7)$ \\
\hline Mean body mass index & $24.1(3.6)$ & $23.8(3.7)$ \\
\hline Premenopausal women (\%) & 40 & 30 \\
\hline Postmenopausal women (\%) & 60 & 70 \\
\hline $\begin{array}{l}\text { History of benign breast } \\
\text { disease }(\%)\end{array}$ & NIL & 31 \\
\hline $\begin{array}{l}\text { Familial history of breast } \\
\text { cancer }(\%)\end{array}$ & NIL & 20 \\
\hline Smokers (\%) & NIL & 1 \\
\hline Alcohol status (\%) & NIL & NIL \\
\hline \multicolumn{3}{|l|}{ Food pattern $(\%)$} \\
\hline Vegetarians & 20 & 40 \\
\hline Mixed diet & 80 & 60 \\
\hline
\end{tabular}

Age and BMI were represented as mean \pm SD. Other characteristics were represented in percentages.

Table 2: FA profile in control women and breast cancer women

\begin{tabular}{llll}
\hline FAs (\%) & Controls (n=30) & Cases $(\mathbf{n = 3 0})$ & p-value \\
\hline Palmitic acid & $7.735(5.2-9.7)$ & $8.26(6.6-10.91)$ & 0.27 \\
Stearic acid & $3.99(2.58-5.31)$ & $3.86(3.19-5.54)$ & 0.69 \\
Palmitoleic & $2.215(1.205-3.155)$ & $3.51(2.33-4.56)$ & $0.005^{* *}$ \\
acid & & & \\
Oleic acid & $11.525(2.49-21.43)$ & $16.67(1.59-24.07)$ & 0.48 \\
LA & $19.9(12.99-27.26)$ & $15.08(9.92-19.89)$ & $0.04^{*}$ \\
GLA & $0.35(0.25-0.74)$ & $0.36(0.27-0.55)$ & 0.88 \\
AA & $3.4(1.45-4.76)$ & $3.45(2.47-6.73)$ & 0.88 \\
DGLA & $2.64(1.46-4.26)$ & $2.47(1.75-3.65)$ & 0.98 \\
Alpha-linolenic & $0.34(0.22-0.45)$ & $0.26(0.21-0.33)$ & 0.3 \\
acid & & & \\
EPA & $1.68(1.05-2.92)$ & $0.99(0.68-1.41)$ & $0.02^{*}$ \\
DPA & $0.62(0.39-1.05)$ & $0.71(0.56-1.04)$ & 0.42 \\
DHA & $1.74(0.91-2.84)$ & $1.42(0.97-2.21)$ & 0.47 \\
Delta 5 & $1.24(0.8-1.72)$ & $1.4(0.72-2.0)$ & 0.72 \\
Desaturase & & & \\
Delta 6 & $0.01(0.0-0.02)$ & $0.01(0.0-0.02)$ & 0.099 \\
Desaturase & & & \\
\hline
\end{tabular}

The FA amount in plasma was c alculated as a percentage of the total FAs. Values were expressed in median and interquartile range $\left(25^{\text {th }}-75^{\text {th }}\right)$. p-value was calculated using the Mann-Whitney U test. ${ }^{* *} \mathrm{p}<0.01,{ }^{*} \mathrm{p}<0.05$. FA: Fatty acid 


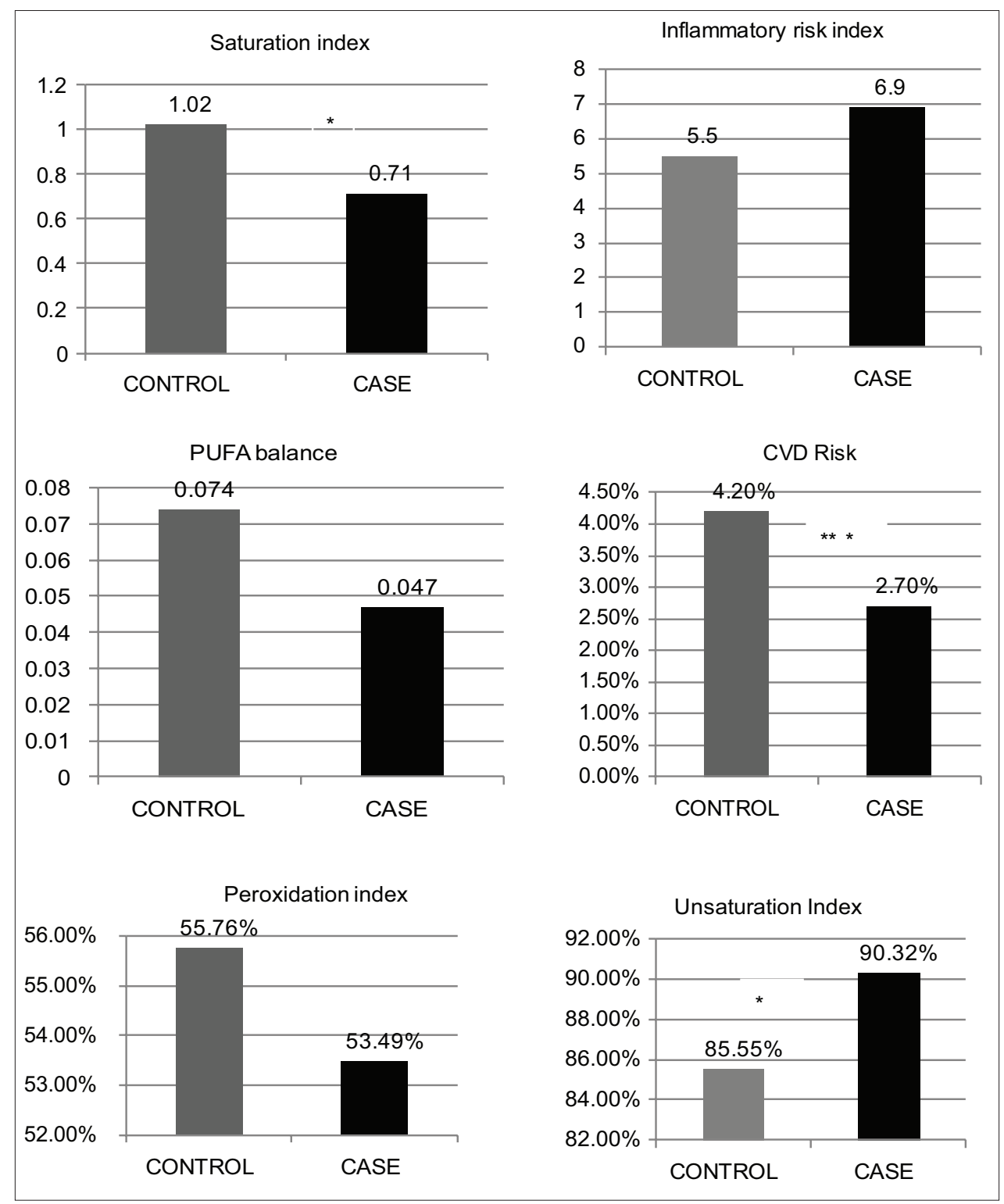

Fig. 1: Molecular biomarkers. Data were expressed as medians. Values that were statistically significantly different are indicated $\left({ }^{*} \mathrm{p}<0.05\right.$, ${ }^{* * *} \mathbf{p}<0.001$ ) based on two-tailed, Mann-Whitney U test

Increased AA and DGLA levels might have contributed to the increased inflammatory risk and decreased PUFA balance in breast cancer women. The increased n-6 FAs produce increased pro-inflammatory 2-series prostaglandins (PGE2) and 4-series leukotrienes, which upsurge inflammation [16,17]. PGE2 is known to induce aromatase expression, thereby increasing breast cancer cell proliferation [19] and also invasion into the lymph nodes by upregulating the chemokine receptors [18].

The inflammatory signaling is important in cancer proliferation pathway. This is sustained by the balance between $n-6$ and n-3 signaling pathway, which emphasizes the importance of dietary omega 3 FAs. FAs related to molecular biomarkers showed differences among the groups. SFA/MUFA ratio was normal in the control group. Cell membrane fluidity has an important role as it serves in the modulation of membrane receptors, ion channels, and transporters [13].

The increased inflammatory risk and CVD risk are due to the lack of FAs or insufficient dietary n-3 FAs. Desaturase enzymatic activity of n-6 and n-3 FAs pathway depends on the availability of FAs and the same enzymes compete for elongation and desaturation. High levels of $n-6$ FAs result in the inhibition of n-3 FAs-derived products [19].
Increased n-3 FAs lead to the production of anti-inflammatory cytokines. EPA and DHA can modify the lipid peroxidation and unsaturation, thereby inhibiting cell oxidative stress and disruption of signaling pathways, leading to tumor transformation [21]. Diet rich in omega 3 FAs inhibits invasion in mammary tumor cells by upregulating the E-cadherin [22], and E-cadherins are important for intracellular adhesion integrity.

Increased desaturation and omega 6 FAs are the important findings in cancer patients, which show the appropriate target for the chemotherapeutical mediations and personalized molecular approach on the lipid profile. Our study concludes that FAs influence the lipidome of the cell membrane and its relevance on the membrane function.

\section{CONCLUSION}

FAs play an important role in cancer growth and proliferation. The present study shows an altered lipid profile in breast cancer women and the potential role of dietary FAs. Using the FA molecular markers, further characterization of cancer patients has to be made on a larger sample with information on the diet. 


\section{AUTHORS' CONTRIBUTIONS}

The first author contributed to all of the writing processes and collection of samples and analysis. The second author contributed to the concept, design, definition of intellectual content, manuscript preparation, editing, and review.

\section{CONFLICT OF INTEREST}

We declare no financial or non-financial interest in subject matter or materials discussed in this manuscript.

\section{REFERENCES}

1. Al-biati HA, Sahib AS, Mahmood AN. Effects of tamoxifen or letrozole on lipid profile, Vitamin D and estradiol serum levels in obese postmenopausal woman with breast cancer. Int J Pharm Pharm Sci 2017;9:141-4

2. Braicu C, Chiorean R, Irimie A, Chira S, Tomuleasa C, Neagoe E, et al., Novel insight into triple-negative breast cancers, the emerging role of angiogenesis, and antiangiogenic therapy. Expert Rev Mol Med 2016;18:e18

3. Larsson SC, Kumlin M, Ingelman-Sundberg M, Wolk A. Dietary longchain n-3 fatty acids for the prevention of cancer: A review of potential mechanisms. Am J Clin Nutr 2004;79:935-45.

4. Liu J, Ma DW. The role of n-3 polyunsaturated fatty acids in the prevention and treatment of breast cancer. Nutrients 2014;6:5184-223.

5. Bassett JK, Hodge AM, English DR, MacInnis RJ, Giles GG. Plasma phospholipids fatty acids, dietary fatty acids, and breast cancer risk. Cancer Causes Control 2016;27:759-73.

6. Hilvo M, Oresie AM. Regulation of lipid metabolism in breast cancer provides diagnostic and therapeutic opportunities. Clin Lipidol 2012;7:177-8

7. Metcalfe LD, Schmitz AA, Pelka JR. Rapid preparation of fatty acid esters from lipids for gas chromatographic analysis. Anal Chem 1966;38:514-5.

8. Amézaga J, Arranz S, Urruticoechea A, Ugartemendia G, Larraioz A, Louka M, et al., Altered red blood cell membrane fatty acid profile in cancer patients. Nutrients 2018;10:E1853.

9. Pironi L, Guidetti M, Verrastro O, Iacona C, Agostini F, Pazzeschi C, et al., Functional lipidomics in patients on home parenteral nutrition: Effect of lipid emulsions. World J Gastroenterol 2017;23:4604-14.

10. Baenke F, Peck B, Miess H, Schulze A. Hooked on fat: The role of lipid synthesis in cancer metabolism and tumour development. Dis Model Mech 2013;6:1353-63.

11. Kumar A, Jha S, Pattanayak SP. Effect of naringenin on lipids, lipoproteins and lipid metabolizing enzymes in 7,12-deimethyl benz(a) anthracene induced mammary carcinogenesis is SD rats. Int J Pharm Pharm Sci 2016;8:154-8.

12. Pamplona R. Membrane phospholipids, lipoxidative damage and molecular integrity: A causal role in aging and longevity. Biochim Biophys Acta 2008;1777:1249-62.

13. Maulucci G, Cohen O, Daniel B, Sansone A, Petropoulou PI, Filou S, et al., Fatty acid-related modulations of membrane fluidity in cells: Detection and implications. Free Radic Res 2016;50:S40-50.

14. Igal RA. Stearoyl coA desaturase-1: New insights into a central regulator of cancer metabolism. Biochim Biophys Acta 2016;1861:1865-80.

15. Pala V, Krogh V, Muti P, Chajès V, Riboli E, Micheli A, et al., Erythrocyte membrane fatty acids and subsequent breast cancer: A prospective Italian study. J Natl Cancer Inst 2001;93:1088-95.

16. Rose DP, Connolly JM. Omega- 3 fatty acids as cancer chemopreventive agents. Pharmacol Ther 1999;83:217-44.

17. Zhou J, Suzuki T, Kovacic A, Saito R, Miki Y, Ishida T, et al., Interactions between prostaglandin $\mathrm{E}(2)$, liver receptor homologue-1, and aromatase in breast cancer. Cancer Res 2005;65:657-63.

18. Pan MR, Hou MF, Chang HC, Hung WC. Cyclooxygenase-2 up-regulates CCR7 via EP2/EP4 receptor signaling pathways to enhance lymphatic invasion of breast cancer cells. J Biol Chem 2008;283:11155-63

19. Simopoulos AP. The importance of the omega-6/omega-3 fatty acid ratio in cardiovascular disease and other chronic diseases. Exp Biol Med (Maywood) 2008;233:674-88

20. Zanoaga O, Jurj A, Raduly L, Cojocneanu-Petric R, Fuentes-Mattei E, $\mathrm{Wu} \mathrm{O}$, et al., Implications of dietary $\omega-3$ and $\omega-6$ polyunsaturated fatty acids in breast cancer. Exp Ther Med 2018;15:1167-76.

21. Dimri M, Bommi PV, Sahasrabuddhe AA, Khandekar JD, Dimri GP. Dietary omega-3 polyunsaturated fatty acids suppress expression of EZH2 in breast cancer cells. Carcinogenesis 2010;31:489-95. 